\title{
Espécies vegetais de uso popular no tratamento da dor: uma revisão sistemática
}

\author{
Plant species of popular use in pain management: a systematic review \\ Especies de plantas de uso popular en el tratamiento del dolor: una revisión sistemática
}

\section{Resumo}

A dor é uma experiência sensitiva e emocional desagradável associada a lesão tecidual real ou potencial; de natureza multifatorial e reconhecida como sério problema de saúde pública. Embora com diversos fármacos sintéticos empregados na terapêutica, é constatado crescente expansão no uso popular de plantas e produtos derivados no tratamento e/ou alívio da dor; situação que merece destaque pela perspectiva do uso popular possibilitar seleção de espécies vegetais para os estudos de validação, mas alertar também para riscos pois a utilização dos insumos vegetais para fins terapêuticos exige certificação de eficácia, segurança e qualidade. Assim, este trabalho objetiva realizar revisão dos estudos etnodirigidos de espécies vegetais utilizadas nas popuçarmente para tratamento da dor. A busca foi realizada nas bases Scielo, LILACS e Pubmed, incluindo artigos publicados de 2011 a 2021, usando como descritores: "dor", "etnobotânica" e "fitoterapia". Foram selecionados 66 artigos, com predomínio de publicações no Brasil e Índia, na língua inglesa; com 70 espécies vegetais referidas, pertencentes a 40 famílias, predominando Asteraceae (12,35\%). As espécies mais referidas foram Curcuma longa L. (7,14\%), Zingiber officinale Roscoe $(5,71 \%)$ e Mentha x piperita (4,28\%); predominando uso das folhas $(54,54 \%)$, preparadas por decocção $(15,15 \%)$ e extração aquosa $(13,63 \%)$. Embora diversas espécies sejam usadas popularmente para tratamento de dor, ainda são escassos os estudos de segurança e eficácia para uso terapêutico. Essa revisão sinaliza espécies a serem selecionadas para prosseguimento com estudo de validação, visando contribuição na oferta de novas opções de tratamento para dor, mas desperta para necessidade de ações de Farmacovigilância em Fitoterapia.

Palavras-chave: Estudo etnodirigido; Estudos de validação; Farmacovigilância; Curcuma longa; Mentha x piperita; Zingiber officinale. 


\begin{abstract}
Abstrac
Pain is an unpleasant sensory and emotional experience associated with actual or potential tissue damage; multifactorial in nature and recognized as a public health problem. Although with synthetic drugs used in therapy, increasing popular use of plants in the treatment and/or relief of pain has been observed; a situation that deserves to be highlighted as it allows selection of plant species for validation studies, but there are risks, as the use of plant-based inputs for therapeutic purposes requires certification of efficacy, safety and quality. Thus, this work aims to carry out a review of ethno-directed studies of plant species used in popular practices for pain treatment. The search was performed in the Scielo, LILACS and Pubmed databases, including articles published from 2011 to 2021, using the descriptors "pain", "ethnobotany" and "phytotherapy". 66 articles were selected, with a predominance of publications in Brazil and India, in English; with 70 plant species mentioned, belonging to 40 families, predominantly the Asteraceae family (12.35\%). The most mentioned plant species were Curcuma longa L. (7.14\%), Zingiber officinale Roscoe (5,71\%) and Mentha x piperita L. (4,28\%); predominating the use of leaves (54.54\%), prepared by decoction $(15.15 \%)$ and aqueous extraction $(13.63 \%)$. Although several plant species are popularly used to treat pain, there are still few studies on safety and efficacy certification for therapeutic use. This review indicates species to be selected for continuation with a validation study, aiming to contribute to the offer of new treatment options for pain, but raises the need for Pharmacovigilance actions in Phytotherapy.
\end{abstract}

Keywords: Ethno-directed study; Validation studies; Pharmacovigilance; Curcuma longa, Mentha x piperita, Zingiber officinale.

\title{
Resume
}

El dolor es una experiencia sensorial y emocional desagradable asociada con daño tisular real o potencial; de naturaleza multifactorial y reconocido como un problema de salud pública. Aunque con las drogas sintéticas utilizadas en terapia, ha habido un uso popular cada vez mayor de plantas en el tratamiento y / o alivio del dolor; situación que merece ser destacada ya que permite la selección de especies vegetales para estudios de validación, pero existen riesgos, ya que el uso de insumos vegetales con fines terapéuticos requiere certificaciones de eficacia, inocuidad y calidad. Así, este trabajo tiene como objetivo realizar una revisión de estudios etnodirigidos de especies vegetales utilizadas en prácticas populares para el tratamiento del dolor. La búsqueda se realizó en las bases de datos Scielo, LILACS y Pubmed, incluyendo artículos publicados entre 2011 y 2021, utilizando los descriptores "dolor", “etnobotánica" y "fitoterapia”. Se seleccionaron 66 artículos, con predominio de publicaciones en Brasil e India, en inglés; con 70 especies vegetales mencionadas, pertenecientes a 40 familias, predominantemente la familia Asteraceae $(12,35 \%)$. Las especies vegetales más mencionadas fueron Curcuma longa L. (7,14\%), Zingiber officinale Roscoe $(5,71 \%)$ y Mentha x piperita L. (4,28\%); el uso predominante de hojas (54,54\%), preparadas por decocción (15,15\%) y extracción acuosa $(13,63 \%)$. Aunque varias especies de plantas se utilizan popularmente para tratar el dolor, todavía hay pocos estudios sobre la certificación de seguridad y eficacia para uso terapéutico. Esta revisión indica especies a ser seleccionadas para continuar con un estudio de validación, con el objetivo de contribuir a la oferta de nuevas opciones de tratamiento para el dolor, pero plantea la necesidad de acciones de Farmacovigilancia en Fitoterapia.

Keywords: Estudio etnodirigido; Estudios de validación; Farmacovigilancia; Curcuma longa, Mentha x piperita, Zingiber officinale.

\section{Introdução}

A dor é definida, segundo Associação Internacional para o Estudo da Dor (IASP), como "uma experiência sensitiva e emocional desagradável associada, ou semelhante àquela associada, a uma lesão tecidual real ou potencial” (Iasp, 2017). Essa definição permaneceu adotada por décadas, porém recentemente o avanço dos estudos, com compreensão mais ampla do fenômeno da dor e seus mecanismos, tem ocasionado mobilização dos especialistas e entidades na revisão conceitual da dor (Desantana \& Perissinotti, 2020).

Estudos mais atualizados dos conceitos de dor incluem: “1. A dor é sempre uma experiência pessoal que é influenciada, em graus variáveis, por fatores biológicos, psicológicos e sociais. 2. Dor e nocicepção são fenômenos diferentes. A dor não pode ser determinada exclusivamente pela atividade dos neurônios sensitivos. 3. Através das suas experiências de vida, as pessoas aprendem o conceito de dor. 4. O relato de uma pessoa sobre uma experiência de dor deve ser respeitado. 5. Embora a dor geralmente cumpra um papel adaptativo, ela pode ter efeitos adversos na função e no bem-estar social e psicológico. 6. A descrição verbal é apenas um dos vários comportamentos para expressar a dor; a incapacidade de comunicação não invalida a possibilidade de um ser humano ou um animal sentir dor" (Raja et al., 2020). 
A dor, aguda ou crônica, ocasiona perda da qualidade de vida, alterações no sono, apetite, libido, disposição; levando a manifestações de irritabilidade, redução na concentração, restrições na capacidade para atividades familiares, incapacidade temporária ou permanente, morbidade; desencadeando impactos pessoais, profissionais e sociais; com elevados custos ao sistema de saúde (Vasconcelos \& Araújo, 2018). Segundo Bettiol et al., (2017), reconhecida como de natureza multifatorial, alguns fatores são associados como: idade, sexo, obesidade, ansiedade, depressão, doenças osteoarticulares, distúrbios do sono e fadiga, hipertensão, diabetes, doenças cardiovasculares e pulmonares.

Embora considerada como sério problema de saúde pública mundial, não há muitos estudos epidemiológicos atuais de prevalência, com estimativa em torno de 10,1 a 55,5\%, com média de 35,5\% da prevalência mundial para dor crônica com 28 a 41\% de prevalência na população brasileira (Posso et al., 2016; Vasconcelos \& Araújo, 2018).

Mesmo diante das diversas estratégias não farmacológicas e farmacológicas para o tratamento da dor, com vasto e diversificado arsenal de fármacos sintéticos, o homem contemporâneo mantem o uso de plantas como alternativa e/ou complemento terapêutico aos sintéticos na terapêutica da dor (Quintans et al., 2014); sendo constatado, nas últimas décadas, crescente expansão no uso de plantas e seus produtos derivados para os mais diversos fins medicinais, especialmente em países de rica biodiversidade, incentivado por diversos fatores, com destaque ao difícil acesso da população aos serviços de saúde, insatisfação com medicamentos sintéticos, legado cultural, modismo e, especialmente, ao estímulo da Organização Mundial de Saúde para adoção de programas e projetos visando valorizar e regulamentar a assistência à saúde pela Fitoterapia (Brasil, 2012; Batalha Júnior et al., 2020; Coutinho et al., 2020).

Mas deve ser enfatizado que a utilização de qualquer insumo de origem vegetal para fins terapêuticos exige a certificação de eficácia, segurança e qualidade do produto disponibilizado ao uso; o que pode ser assegurado por um conjunto de medidas, com esforços e recursos direcionados para o desenvolvimento dos estudos de validação (Hasenclever et al., 2017).

A validação de espécies vegetais envolve estudos etnodirigidos, botânicos, agronômicos, químicos, biológicos e farmacêuticos; possibilitando a investigação científica das propriedades terapêuticas empiricamente atribuídas às plantas pelo uso popular, visando emprego como medicamento em seres vivos; representando, assim, o único caminho para transformar as plantas em produtos fitoterápicos (Simões et al., 2017; Amaral et al., 2021).

No entanto, o desenvolvimento desses estudos lida com uma pergunta inicial: em um país de rica biodiversidade e diversidade cultural como o Brasil, o que vale ser selecionado para alvo dessas investigações? Nesse sentido, os estudos etnodirigidos (etnobotânicos e etnofarmacológicos) fornecem importantes subsídios, possibilitando a avaliação dos recursos naturais, especialmente de origem vegetal, empregados terapeuticamente pela população, pois o "saber popular" pode ser encarado como pré-triagem quanto à utilidade terapêutica (Albuquerque et al., 2009; Oliveira et al., 2009; Amaral et al., 2021). Mas deve ser enfatizado que o uso popular e tradicional não é suficiente para validar plantas como medicamentos; sendo assim, os dados da pesquisa etnodirigida devem ser empregados como critério de seleção de espécies vegetais alvo para prosseguimento dos estudos de validação (Albuquerque et al., 2014; Amaral et al., 2021).

Além do reconhecimento dos estudos etnodirigidos representarem a ferramenta mais robusta na difícil etapa de selecionar material para continuidade dos estudos de validação, o uso popular de plantas para fins terapêuticos alicerçada no mito "se natural não faz mal", sem comprovação da eficácia, espectro toxicológico e garantia de qualidade, expõem a população a riscos dado o uso irracional (Silveira et al., 2008; Tovar \& Petzel, 2009; Godinho, 2014; Campos et al., 2016; Amaral et al., 2021); exigindo ações de Farmacovigilância em Fitoterapia, com incentivo a adoção de medidas visando minimizar eventos adversos associados ao uso irracional de plantas para fins terapêuticos (Batalha Júnior et al., 2020; Silva et al., 2021).

Assim, na perspectiva de fornecer subsídios para definir critérios de seleção de espécies vegetais para prosseguimento dos estudos de validação com ênfase no tratamento e/ou alívio da dor; bem como identificar potencialidade de 
toxicidade no uso popular de tais plantas; este trabalho foi desenvolvido com objetivo de realizar uma revisão dos estudos etnodirigidos de espécies vegetais utilizadas nas práticas populares para tratamento da dor.

\section{Metodologia}

De acordo com Galvão e Pereira (2014) O estudo é uma revisão sistemática da literatura, com 05 (cinco) etapas norteadoras adotadas para realização do trabalho:

a) Elaboração do conceito principal norteador da pesquisa: verificar as publicações acadêmicas nos últimos 10 anos (2011 - 2021) sobre uso popular de espécies vegetais no tratamento da dor;

b) Busca ou amostragem na literatura de trabalhos etnodirigidos relacionados ao tema, em concordância aos critérios de inclusão e exclusão definidos;

c) Coleta de dados;

d) Análise crítica dos trabalhos relevantes;

e) Apresentação e discussão dos resultados.

A busca foi realizada nas bases de dados eletrônicas: Literatura Latino-Americana do Caribe em Ciências da Saúde (LILACS), National Library of Medicine and National Institutes of Health (PUBMED) e Scientific Eletronic Library Online (SCIELO). As referências que preencheram os critérios de inclusão foram avaliadas com relação ao conteúdo. A seleção dos descritores utilizados no processo de revisão foi efetuada mediante consulta a base "Descritores de Assunto em Ciências da Saúde da BIREME (DECs)”. Nas buscas, os seguintes descritores, em língua portuguesa e inglesa, foram considerados: "dor" (descritor 1); "etnobotânica" (descritor 2); "fitoterapia" (descritor 3).

Foram utilizadas etapas e descritores em categorias para a seleção dos artigos: análise nos bancos de dados com 04 (quatro) categorias de descritores em arranjos, sendo utilizados: "dor", "etnobotânica" e "fitoterapia"; dispostos da seguinte forma: "dor" e "etnobotânica"; "dor" e "fitoterapia"; "etnobotânica" e "fitoterapia"; "dor" e "etnobotânica" e "fitoterapia".

Para a seleção da amostra foram aplicados os seguintes critérios: a) Inclusão: trabalhos publicados de 2011 a 2021, com abordagem do tema proposto e publicados em inglês, português ou espanhol; $b$ ) Exclusão: artigos que não se enquadram em "open access", trabalhos que fugiram ao tema apesar de conter os descritores, publicações sob forma de reportagens e capítulos de livros, cartas ao editor, teses, dissertações e monografias.

Para extrair os dados dos artigos selecionados foi utilizado um formulário resumido que sumariza aspectos referentes aos autores, título, ano de publicação, planta usada/analisada no estudo, parte da planta e tipo de preparação; com dados trabalhados de forma sistemática através da ordenação, classificação e análise final do conteúdo dos artigos, seguida da etapa de tabulação dos mesmos.

\section{Resultados e Discussão}

Com as combinações de descritores, segundo metodologia adotada no desenvolvimento dessa revisão, foram préselecionados 745 artigos disponibilizadas nas bases de dados (Tabela 1). 
Tabela 1. Número de artigos conforme arranjos dos descritores usados na pesquisa em base de dados da revisão sistemática do uso de espécies vegetais no tratamento e/ou alívio da dor, 2011 a 2021.

\begin{tabular}{llll}
\hline COMBINAÇÕES & LILACS & PUBMED & SCIELO \\
\hline "dor" E "etnobotânica" & 12 & 30 & 0 \\
\hline "dor" E "fitoterapia" & 25 & 438 & 7 \\
\hline "etnobotânica" E "fitoterapia" & 3 & 216 & 1 \\
\hline "dor", "etnobotânica" E "fitoterapia" & 2 & 11 & 0 \\
\hline Total & $\mathbf{4 2}$ & $\mathbf{6 9 5}$ & $\mathbf{8}$ \\
\hline
\end{tabular}

Fonte: Autores. Dados obtidos nas bases de dados LILACS, PUBMED e SCIELO.

Após a análise, eliminando a duplicidade dos artigos disponibilizados concomitantemente em 02 (duas) ou mais das bases de dados, foi obtida amostra final de 65 artigos, que atenderam à questão norteadora do estudo e critérios de inclusão e exclusão; predominando publicações em língua inglesa (n: 53), nos anos de 2012 (16,92\%) e 2017 (13,84\%) (Figura 1).

Figura 1. Distribuições de trabalhos, por ano, em números absolutos, das publicações disponibilizadas nas bases de dados da revisão sistemática do uso de espécies vegetais no tratamento e/ou alívio da dor, 2011 a 2021.

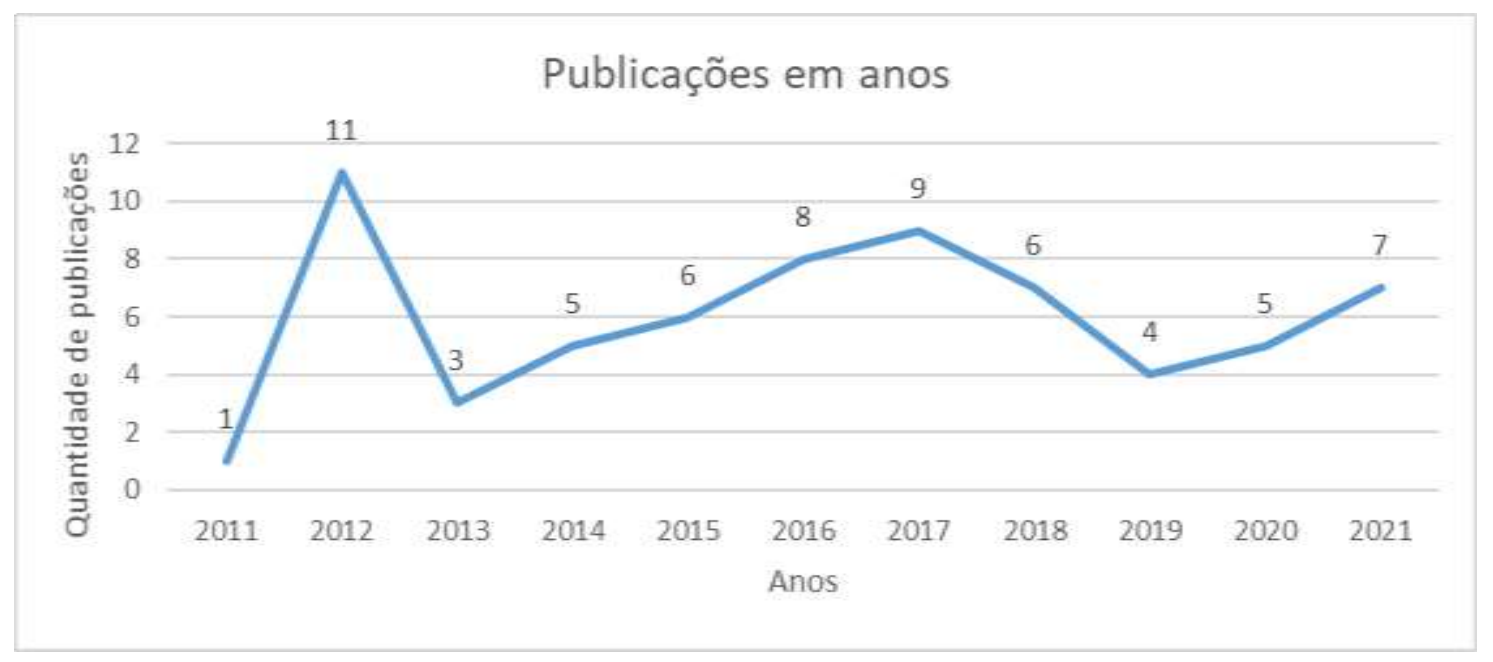

Fonte: Autores. Dados obtidos nas bases de dados LILACS, PUBMED e SCIELO.

Com relação aos países de origem da publicação, predominaram publicações no Brasil (23,18\%) e Índia (11,59\%); seguido da Etiópia (4,34\%), Paquistão (4,34\%) e África do Sul (2,89\%). A predominância dos estudos publicados no Brasil pode refletir tendência do amplo consumo de recursos naturais, com ênfase aos de origem vegetal, dado a grande biodiversidade, legado cultural e desigualdades econômicas com grande parte da população sem acesso aos serviços e bens de saúde, favorecendo o uso popular de plantas para fins terapêuticos, estimulando os estudos nessa área (Batalha Júnior et al., 2020; Coutinho et al., 2020; Amaral et al., 2021).

Em relação a parte do vegetal empregada nas preparações populares (Tabela 2), evidenciamos que predomina uso de órgãos vegetais isolados: folhas $(54,54 \%)$, raízes $(15,15 \%)$, frutos $(12,12 \%)$, flores $(6,06 \%)$, cascas do caule $(6,06 \%)$, caules $(3,03 \%)$ e sementes $(3,03 \%)$. Estudos etnodirigidos locais visando caracterização do uso popular de plantas para mais diversos fins terapêutico, nas várias faixas etárias, quer em zona rural ou urbana, constatam predomínio do uso das folhas nas preparações terapêuticas caseiras; o que pode ser justificado pela facilidade de acesso e tradicionalidade em algumas culturas (Tugume \& Nyakoojo, 2019; Novotna et al., 2020). 
Quanto as preparações, predomina uso de decocção (20,73\%), extrato aquoso (10,97\%), seguida por hidralcóolico (3,65\%); enfatizando que 52,43\% dos trabalhos analisados não

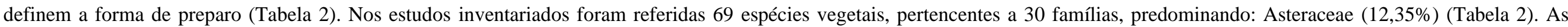
espécies vegetais mais referidas foram: Curcuma longa L. (7,14\%), Zingiber officinale Roscoe (5,14 \%) e Mentha x piperita L. (4,28\%) (Tabela 2).

Tabela 2. Estudos inventariados na revisão de espécies vegetais empregadas popularmente no tratamento e/ou alívio da dor, 2011 a 2021.

\begin{tabular}{|c|c|c|c|c|c|c|}
\hline FAMÍLIA & ESPÉCIE & PARTE DA PLANTA & PREPARO & PAÍS & $\begin{array}{c}\text { MODELO } \\
\text { DE } \\
\text { ESTUDO }\end{array}$ & REFERÊNCIA \\
\hline \multirow[t]{2}{*}{ Acanthaceae } & Justicia adhatoda L. & folhas & decocção & Paquistão & Etnobotânico & Malik et al. (2019) \\
\hline & Justicia pectoralis Jacq & folhas & decocção & Brasil & Etnobotânico & Pereira et al. (2015) \\
\hline \multirow{3}{*}{ Amaranthaceae } & Alternanthera tenella Colla & NI & decocção & Brasil & Etnobotânico & Magalhães et al. (2021) \\
\hline & Alternanthera brasiliana $\mathrm{L}$. & folhas & $\mathrm{NI}$ & Brasil & Etnobotânico & $\begin{array}{l}\text { Dresch; Liborio; } \\
\text { (2021) }\end{array}$ \\
\hline & Chenopodium ambrosioides $\mathrm{L}$. & raízes & NI & Brasil & Etnobotânico & Pedrollo et al. (2016) \\
\hline \multirow[t]{2}{*}{ Anacaediaceae } & \multirow{2}{*}{ Schinus molle $\mathrm{L}$. } & $\begin{array}{l}\text { raízes, planta inteira, sementes, cascas } \\
\text { de caule, folhas }\end{array}$ & NI & África do Sul & Etnobotânico & Maema et al. (2016) \\
\hline & & folhas & infusão & Bolívia & Etnobotânico & $\begin{array}{l}\text { Quiroga; } \text { Meneses; } \quad \text { Bussmann } \\
\text { (2012) }\end{array}$ \\
\hline Apiaceae & Heracleum persicum $\mathrm{L}$. & sementes & NI & Noruega & Etnobotânico & $\operatorname{Alm}(2013)$ \\
\hline \multirow[t]{2}{*}{ Apocynaceae } & Rhazya stricta Decne & folhas, cascas de caule & decocção & Irã & Etnobotânico & Hosseini et al. (2021) \\
\hline & Calotropis procera Aiton & folhas, cascas de caule & decocção & Irã & Etnobotânico & Hosseini et al. (2021) \\
\hline Araceae & Colocasia esculenta $\mathrm{L}$. & NI & NI & Bangladesh & Etnobotânico & Islam et al. (2020) \\
\hline \multirow{10}{*}{ Asteraceae } & Artemisia аппиа $\mathrm{L}$. & folhas & extrato aquoso & China & Ensaio clínico & Yang et al. (2014) \\
\hline & Solidago chilensis Meyen & folhas & extrato glicólico & Brasil & Ensaio clinico & Silva et al. (2015) \\
\hline & Adenostemma sp. & $\begin{array}{l}\text { flores, frutos ou nozes, galhos com } \\
\text { folhas }\end{array}$ & decocção & Nova Guiné & Etnobotânico & Jorim et al. (2012) \\
\hline & Artemisia scoparia Waldst & folhas, caules, cascas de caule & NI & Paquistão & Etnobotânico & Ahmad et al. (2017) \\
\hline & Ageratum conyzoides $\mathrm{L}$. & folhas, caules, cascas de caule & NI & Paquistão & Etnobotânico & Ahmad et al. (2017) \\
\hline & \multirow{2}{*}{ Matricaria chamomilla $\mathrm{L}$. } & flores & óleo essencial & Paquistão & Ensaio randomizado & Shora et al. (2015) \\
\hline & & flores & $\mathrm{NI}$ & Pérsia & Estudo randomizado & Zargaran et al. (2018) \\
\hline & \multirow{3}{*}{ Vernonia amygdalina Delile } & NI & NI & Uganda & Etnobotânico & Tabuti et al. (2012) \\
\hline & & folhas & decocção & Uganda & Etnobotânico & Tugume et al. (2016) \\
\hline & & NI & NI & Uganda & Etnobotânico & Tabuti et al. (2012) \\
\hline Basellaceae & Anredera cordifolia $($ Tem.) Steenis & frutos & NI & Brasil & Etnobotânico & Medeiros et al. (2021) \\
\hline
\end{tabular}


Research, Society and Development, v. 11, n. 2, e22511225608, 2022

(CC BY 4.0) | ISSN 2525-3409 | DOI: http://dx.doi.org/10.33448/rsd-v11i2.25608

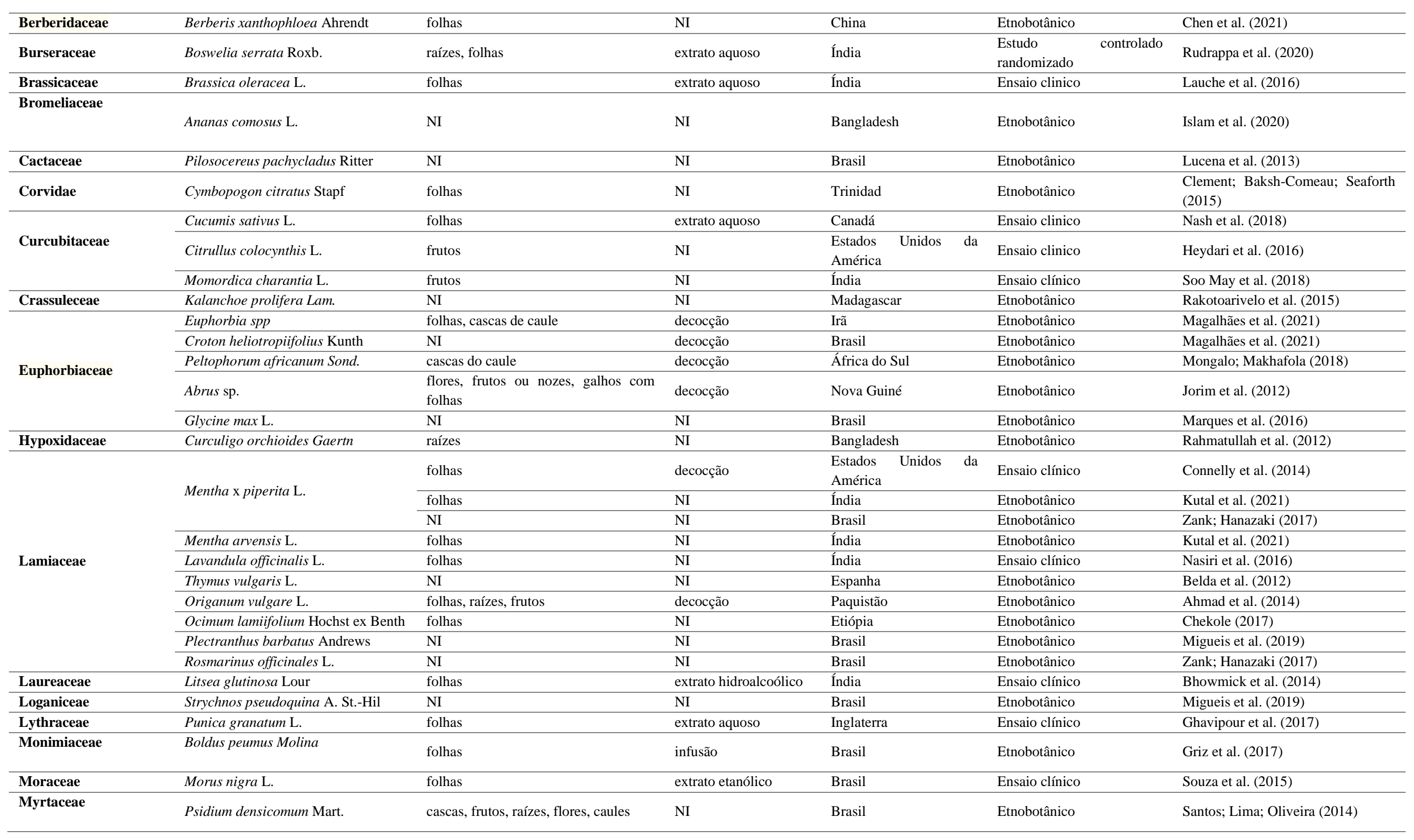


Research, Society and Development, v. 11, n. 2, e22511225608, 2022

(CC BY 4.0) | ISSN 2525-3409 | DOI: http://dx.doi.org/10.33448/rsd-v11i2.25608

\begin{tabular}{|c|c|c|c|c|c|c|}
\hline Nepetoideae & Plectranthus barbatus Andr. & folhas & decocção & Brasil & Etnobotânico & Pereira et al. (2015) \\
\hline Orchidaceae & Vanda tessellata (Roxb.) Hook & folhas, raízes & maceração & Bangladesh & Etnobotânico & Rahmatullah et al. (2012) \\
\hline Papaveraceae & $\begin{array}{l}\text { Papaver somniferum } \\
\text { L. }\end{array}$ & frutos & NI & Brasil & Etnobotânico & Medeiros; Albuquerque (2012) \\
\hline Piperaceae & Piper cavalcantei Yunck & cascas, frutos, raízes, flores, caules & NI & Brasil & Etnobotânico & Santos; Lima; Oliveira (2014) \\
\hline Plantaginaceae & Plantago major $\mathrm{L}$. & folhas, flores, raízes & decocção & Turquia & Etnobotânico & $\begin{array}{l}\text { Kazanci; Oruç; Mosulishvili } \\
(2020)\end{array}$ \\
\hline Poaceae & Cymbopogon citratus Stapf. & NI & NI & Brasil & Etnobotânico & Migueis et al. (2019) \\
\hline \multirow{4}{*}{ Rutaceae } & Citrus sinensis $\mathrm{L}$. & NI & NI & Brasil & Etnobotânico & Migueis et al. (2019) \\
\hline & Aegle marmelos $\mathrm{L}$. & NI & NI & Bangladesh & Etnobotânico & Islam et al. (2020) \\
\hline & $\begin{array}{l}\text { Ruta graveolens } \\
\text { L. }\end{array}$ & NI & NI & Brasil & Etnobotânico & Zank; Hanazaki (2017) \\
\hline & $\begin{array}{l}\text { Pilocarpus microphyllus } \\
\text { Stapf. }\end{array}$ & cascas, frutos, raízes, flores, caules & NI & Brasil & Etnobotânico & Santos; Lima; Oliveira (2014) \\
\hline Solanaceae & Capsicum annuит $\mathrm{L}$. & frutos & NI & Índia & Ensaio clínico & Musharraf et al. (2017) \\
\hline Tamaricaceae & Tamarix galica $\mathrm{L}$. & NI & NI & Itália & Etnobotânico & Montesano (2012) \\
\hline Theaceae & Camellia sinensis $\mathrm{L}$. & folhas & decocção & Índia & Estudo clínico & Hashempur et al. (2018) \\
\hline \multirow{3}{*}{ Verbenaceae } & Lippia sidoides Cham. & folhas & decocção & Brasil & Etnobotânico & Pereira et al. (2015) \\
\hline & \multirow{2}{*}{ Lippia alba (Mill.) NE Brown } & folhas & extrato hidroetanólico & Brasil & Ensaio clínico & Carmona et al. (2013) \\
\hline & & folhas & extrato hidroalcoólico & Brasil & Ensaio clínico & Conde et al. (2011) \\
\hline Vitaceae & $\begin{array}{l}\text { Cyphostemma adenocaule (Steud ex } \\
\text { A. Rich) }\end{array}$ & NI & NI & Etiópia & Etnobotânico & $\begin{array}{l}\text { Zeynu; Wondimu; } \quad \text { Demissew } \\
(2021)\end{array}$ \\
\hline \multirow{9}{*}{ Zingiberaceae } & \multirow{5}{*}{ Curcuma longa $\mathrm{L}$. } & folhas & extrato aquoso & Austrália & Ensaio clínico & Wang et al. (2020) \\
\hline & & raízes, folhas & extrato aquoso & Índia & Estudo clínico & Rudrappa et al. (2020) \\
\hline & & folhas & extrato aquoso & Índia & Ensaio clínico & Kuptniratsaikul et al. (2014) \\
\hline & & rizomas & óleo essencial & $\begin{array}{lll}\text { Estados } & \text { Unidos } & \text { da } \\
\text { América } & & \end{array}$ & Ensaio clínico & Shep et al. (2020) \\
\hline & & raízes, folhas & infusão, decocção & Nepal & Etnobotânico & Singh et al. (2012) \\
\hline & Zingiber officinale Roscoe & folhas & extrato aquoso & China & Ensaio clínico & Amorndoljai et al. (2017) \\
\hline & & folhas & pulverização & Tailândia & Ensaio clínico & Niempoong et al. (2012) \\
\hline & & raízes & in natura & Paquistão & Ensaio clínico & Mozaffari-Khosravi et al. (2016) \\
\hline & & folhas, raízes & maceração & Bangladesh & Etnobotânico & Rahmatullah et al. (2012) \\
\hline
\end{tabular}

NI: Não Informado Fonte: Autores. 
Diversas classes de metabólitos secundários comprovadamente são eficazes na modulação da dor, contribuindo significativamente no arsenal terapêutico para tratamento e/ou alívio da dor, a exemplo de morfina, canabidiol e ácido acetilsalicílico (Singla et al., 2021). Estudo de revisão de Quintans et al. (2014) refere importantes classes de metabólitos secundários no controle da dor, tais como: a) flavonoides atuando por meio da sua atividade antioxidante e pela modulação da atividade da proteína C quinase; b) terpenos com atuação no Sistema Nervoso Central modulando neurotransmissores, com papel importante na modulação da dor crônica, a exemplo da dor neuropática; c) alcaloides, especialmente os indólicos.

Estudo de revisão de Uddin et al. (2020) também enfatiza o papel dos flavonoides, especialmente nas dores neuropáticas, atuando por diferentes mecanismos, como inibindo a redução da defesa antioxidante, diminuindo o estresse oxidativo, inibindo a hiperativação de PARP, inibindo a lesão celular e os processos de disfunção mitocondrial e inibindo a ativação das células gliais e neuroinflamação. O potencial antinociceptivo de óleos essenciais é enfatizado em revisão de Assis et al. (2020).

Curcuma longa, Mentha x piperita e Zingiber officinale representam as espécies vegetais com mais referências de uso popular em dor constatadas nessa revisão. Essas espécies estão inclusas no Formulário de Fitoterápicos da Farmacopeia Brasileira (Brasil, 2021), mas avaliando as indicações terapêuticas disponibilizadas nesse compêndio oficial, constatamos que não há referências ao uso de tais espécies no tratamento e/ou alívio da dor; porém as presenças de metabólitos secundários comprovadamente eficazes na modulação da dor em tais espécies, a exemplo dos flavonoides, devem estimular os estudos de validação em modelos in vitro e in vivo de analgesia.

Mas vale enfatizar que tais estudos de validação devem ser fundamentados na investigação da eficácia terapêutica dos extratos vegetais e suas preparações derivadas na analgesia, incluindo também os parâmetros de segurança ao uso. Nesse contexto destacamos que, dentre as espécies vegetais referidas de uso popular na dor e com classes de metabólitos secundários que indicam potencial, já existem trabalhos publicados em compêndios oficiais com evidências de eventos adversos, tais como intoxicações e interações medicamentosas (Tabela 3).

Tabela 3. Eventos adversos atribuídos a espécies vegetais empregadas popularmente no tratamento da dor, referidas em estudos etnobotânicos inventariados, 2011 a 2021.

\begin{tabular}{|c|c|}
\hline ESPÉCIE VEGETAL & EVENTOS ADVERSOS \\
\hline $\mathrm{Cu}$ & $\begin{array}{l}\text { Interações com antiagregantes plaquetários, anticoagulante, anti-inflamatórios não esteroidais, imunossupressores, } \\
\text { paclitaxel, irinotecán, oseltamivir, cotrimoxazol (Marchi et al., 2016; Aché, 2020) e produtos antioxidantes (Brasil, 2021). } \\
\text { Contraindicação em portadores de cálculos biliares (WHO, 1999), úlcera gastroduodenal e hiperacidez estomacal (Brasil, } \\
\text { 2015a; Brasil, 2021). Evidências de hepatoxicidade, genotoxicidade, alteração de peso, aumento no número de eritrócitos } \\
\text { policromáticos micronucleados e nas frequências de aberrações cromossômicas totais (Brasil, 2015). Consumo associado } \\
\text { ao paracetamol pode aumentar toxicidade devido à indução de CYP1A2 (Brasil, 2015). }\end{array}$ \\
\hline Mentha $x$ piperita $\mathrm{L}$. & $\begin{array}{l}\text { Interações com medicamentos repositores de estrogênio, potencializando os efeitos; inibição do metabolismo de fármacos } \\
\text { metabolizados por subtipos de CYP3A; aumenta os efeitos de fármacos inibidores do canal de cálcio ou outros } \\
\text { hipotensores cronotrópicos negativos, podendo reduzir a absorção de ferro (Brasil, 2021). Contraindicado em pacientes } \\
\text { com distúrbios gastrointestinais, comprometimento hepático (WHO, 2002), da vesícula biliar (WHO, 2002; Brasil, 2021), } \\
\text { obstrução dos ductos biliares, danos hepáticos severos e litíase urinária (Brasil, 2021). Há relatos de queixas gástricas, } \\
\text { erupções cutâneas, dor de cabeça, azia, queimação perianal, bradicardia, tremores musculares e ataxia como efeitos } \\
\text { colaterais (WHO, 2002), lesões hepáticas, nefrite intersticial e insuficiência renal aguda (Brasil, 2021). }\end{array}$ \\
\hline $\begin{array}{l}\text { Zingiber } \\
\text { Roscoe }\end{array}$ & $\begin{array}{l}\text { Interações com ácido acetilsalicílico, varfarina, heparina, clopidogrel, ibuprofeno, naproxeno ou outros medicamentos que } \\
\text { apresentem ação anticoagulante (WHO, 1999; Abebe, 2002; Nicoletti et al., 2007; Ali et al., 2008; Cardoso et al., 2009; } \\
\text { Nicoletti et al., 2010; Dias et al., 2017), medicamentos gastroprotetores (WHO, 1999; Nicoletti et al., 2007; Nicoletti et al., } \\
\text { 2010). Contraindicado em pacientes com cálculos biliares, distúrbios metabólicos como hipertensão e diabete (Brasil, } \\
\text { 2021). Possui ação inibidora da ciclooxigenase e 5-lipoxigenase (Brasil, 2021). }\end{array}$ \\
\hline
\end{tabular}


No alerta aos riscos associados ao uso popular de plantas para qualquer fim terapêutico, especialmente no tratamento e/ou alivio da dor dada alta ocorrência, merece enfatizar as contraindicações e restrições de uso durante a gestação, lactação e em crianças, especialmente menores de 06 anos, dada falta de parâmetros de segurança (Brasil, 2021). Vale também enfatizar os riscos da prática popular de preparações com associações de espécies vegetais, pela potencialidade de ocorrer interação das plantas com sinergismo dos seus bioativos podendo ocasionar efeitos tóxicos (Casanova; Costa 2017).

Assim, embora plantas e seus produtos derivados sejam tradicionalmente empregados popularmente para o tratamento, cura e prevenção de diversas afecções, agudas e crônicas, a exemplo da dor como evidenciado nessa revisão; essa tendência de uso, principalmente estimulada pela crença errônea de ausência de eventos adversos no uso de produtos naturais, alicerçada no mito "se natural, não faz mal", merece atenção e ações efetivas da Farmacovigilância em Fitoterapia, como subsídio na educação em saúde e promoção do uso seguro e racional (Batalha Junior et al., 2020).

\section{Conclusão}

A revisão dos estudos etnobotânicos que fundamentam esse trabalho evidencia diversidade de espécies vegetais empregadas na prática popular do tratamento e/ou alivio da dor. Curcuma longa, Mentha x piperita e Zingiber officinale, espécies vegetais mais referidas nessa revisão, representam espécies inclusas no Formulário de Fitoterápicos da Farmacopeia Brasileira (Brasil, 2021), porém sem estudos que comprovem eficácia terapêutica na dor; mas com constatação de toxicidade, interações medicamentosas e contraindicações ao uso. Para futuros trabalhos com a mesma situação devem ser estimulado a continuidade dos estudos de validação com tais espécies, pois ainda precisa maiores conhecimentos sobre a eficácia das plantas para tratamento da dor.

Nesse sentido constatamos a abrangência dos estudos etnodirigidos, com ênfase aos etnobotânicos e etnofarmacológicos, contribuindo efetivamente no difícil processo de seleção de espécies vegetais para estudos de validação, mas também para nortear ações efetivas de Farmacovigilância, na perspectiva real de minimizar riscos associados ao uso popular de plantas para fins medicinais.

\section{Agradecimentos}

Os autores agradecem a Coordenação de Aperfeiçoamento de Pessoal de Nível Superior (CAPES), a Fundação de Amparo à Pesquisa e ao Desenvolvimento Científico e Tecnológico do Estado do Maranhão (FAPEMA) e ao Conselho Nacional de Desenvolvimento Científico e Tecnológico (CNPQ) pelo apoio financeiro (Código de Financiamento 001).

\section{Referências}

Abebe, W. (2002). Herbal medication: potential for adverse interactions with analgesic drugs. Journal of Clinical Pharmacy and Therapeutics. 27, 391-401

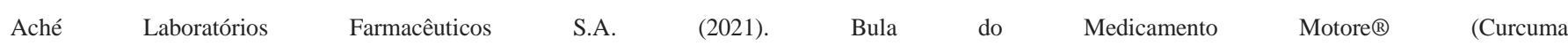
longa). http://www.ache.com.br/Downloads/BulaRapida.aspx?ProductId=445/.

Ahmad, K. S., Hamid, A., Nawaz, F., Hameed, M., Ahmad, F., Deng, J., Akhtar, N., Wazarat, A. \& Mahroof, S. (2017). Ethnopharmacological studies of indigenous plants in Kel village, Neelum Valley, Azad Kashmir, Pakistan. Journal of Ethnobiology and Ethnomedicine. 13(68). 10.1186 / s13002-017-0196-1

Albuquerque, U. P., Fonseca-Krueliv, V. S. \& Hanazaki, N. (2009). Avanços nas pesquisas etnobotânicas no Brasil. Acta Botanica Brasilica. $23(2)$, 590-605.

Albuquerque, U. P., Medeiros, P. M., Ramos, M. A., Ferreira Júnior, W. S., Nascimento, A. L. B., Avilez, W. M. T., \& Melo, J. G. (2014). Are ethnopharmacological surveys useful for the discovery and development of drugs from medicinal plants? Revista Brasileira de Farmacognosia, 24,110 -115.

Ali Hussain, H. E. (2002). Hypoglycemic, hypolipidemic and antioxidant properties of combination of Curcumin from Curcuma longa, Linn, and partially purified product from Abroma augusta, Linn. in streptozotocin induced diabetes. Indian Journal of Clinical Biochemistry, $17(2), 33$ - 43.

Alm, T. (2013). Ethnobotany of Heracleum persicum Desf. ex Fisch., an invasive species in Norway, or how plant names, uses, and other traditions evolve.Journal of Ethnobiology and Ethnomedicine, 9, 42, 10.1186/1746-4269-9-42. 
Amaral, F. M. M., Oliveira, M. A., Coutinho, D. F., Godinho, J. W. L. S., Cartágenes, M. S. S., Neiva, V. A., Neiva Neto, R. R., \& Bastos, W. M. (2021). Estudo de validação de espécies vegetais: o elo entre o saber popular e o fitoterápico. In: Trajetória e pesquisa nas ciências farmacêuticas. 1 ed. Ponta Grossa: Atena Editora, 1, 33 - 54 .

Amorndoljai, P., Taneepanichskul, S., Niempoog, S., \& Nimmannit, U. A (2017). Comparative of ginger extract in nanostructure lipid carrier (NLC) and 1\% diclofenac gel for treatment of knee osteoartritis (OA). Journal of the Medical Association of Thailand, 100 (4), $447-456$.

Assis, D. B., Aragão Neto, H. C., Fonsêca, D. V., Andrade,H. H. N., Braga, R. M., Badr, N., Maia, M. S., Castro, R. D., Scotti, L., Scotti, M. T., \& Almeida, R. N. (2020). Antinociceptive activity of chemical components of essential oils that involves docking studies: a review. Frontiers in Pharmacology, (29). https://doi.org/10.3389/fphar.2020.00777

Batalha Júnior, N. J. P, Amaral, F. M. M., Costa, I. S., Batalha, M. N., Coutinho, D. F., Godinho, J. W. S., Britto, M. H. S. S., \& Bezerra, S. A. (2020). FARMACOVIGILÂNCIA EM FITOTERAPIA: uma breve abordagem. In: Ciências da Saúde no Brasil: Impasses e Desafios 2. 1 ed. Ponta Grossa: Atena Editora, 2, $62-76$.

Belda, A., Zaragozí, B., Belda, I., Martínez, J., \& Seva, E. (2012). Traditional knowledge of medicinal plants in the Serra de Mariola Natural Park, Southeastern Spain. African Journal of Traditional, Complementary and Alternative Medicines, 10(2), 299 - 309. 10.4314/ajtcam.v10i2.15.

Bettiol, C., Dellaroza, M., Lebrão, M. L., Duarte, Y. A., \& Santos, H. (2017). Fatores preditores de dor em idosos do Município de São Paulo, Brasil: Estudo SABE 2006 e 2010. Cadernos de Saúde Pública, 33, (9), e00098416. https://doi.org/10.1590/0102- 311X00098416.

Bhowmick, R., Sarwa, M. S., Dewan, S. M. R., Das, A., Das, B., Uddin, M. M. S., Islam, M. S., \& Islam, M. S. (2014). In vivo analgesic, antipyretic, and antiinflammatory potential in Swiss albino mice and in vitro thrombolytic activity of hydroalcoholic extract from Litsea glutinosa leaves. Biological Research, 47 , $1-8$.

Brasil. (2015). Monografia da espécie Curcuma longa L. (curcuma). Ministério da Saúde. 158p.

Brasil. (2021). Agência Nacional de Vigilância Sanitária. RDC no 463, de 27 de janeiro de 2021.2 ed. Formulário de Fitoterápicos da Farmacopéia Brasileira. Agência Nacional de Vigilância Sanitária. Brasília: Anvisa, 223.

Brasil. (2012). Práticas integrativas e complementares: plantas medicinais e fitoterapia na Atenção Básica. Ministério da Saúde. Secretaria de Atenção à Saúde. Departamento de Atenção Básica Brasília, 156.

Campos, S. C., Silva, C. G., Campana, P. R. V., \& Almeida, V. L. (2016). Toxicidade de espécies vegetais. Revista Brasileira de Plantas Medicinais, 18, 373 -382 .

Cardoso, C. M. Z., Silva, C. P., Yamagami, K., Lopes, R. P., Santos, F., Bossani, I., Jesuíno, I., Geres, F., Martorie Junior., T., Graça, M., Kaneko, B., Pavani, E., \& Inowe, C. (2009). Elaboração de uma cartilha direcionada aos profissionais da área da saúde, contendo informações sobre interações medicamentosas envolvendo fitoterápicos e alopáticos. Revista Fitos, 4(1), 56 - 69.

Carmona, F., Angelucci, M. A., Sales, D. S., Chiaratti, T. M., Honorato, F. B., Bianchi, R. V., \& Pereira, A. M. (2013). Lippia alba (Mill.) N. E. Brown hydroethanolic extract of the leaves is effective in the treatment of migraine in women. Fitomedicina. 20(10), 947-950. 10.1016/ j.phymed.2013.03.017.

Casanova, L. M., \& Costa, S. S. (2016). Interações sinérgicas entre plantas medicinais e medicamentos. Revista Saúde e Desenvolvimento, $9(5), 4$-19.

Chekole, G. (2017). Ethnobotanical study of medicinal plants used against human ailments in Gubalafto District, Northern Ethiopia. Journal of Ethnobiology and Ethnomedicine, 13(55). 10.1186/s13002-017-0182-7.

Chen, W. Y., Yang, T., Yang, J., Qiu, Z. C., Ding, X. Y., Wang, Y. H., \& Wang, Y. H. (2021). Wild plants used by the Lhoba people in Douyu Village, characterized by high mountains and valleys, in southeastern Tibet, China. Journal of Ethnobiology and Ethnomedicine, 17, (46). 10.1186/s13002-021-00472-

Clement, Y. N., Baksh-Comeau, Y. S., \& Seaforth, C. E. (2015). An ethnobotanical survey of medicinal plants in Trinidad. Journal of Ethnobiology and Ethnomedicine, 11(67). 10.1186/s13002-015-0052-0.

Conde, R., Corrêa, V. S., Carmona, F., Contini, S. H., \& Pereira, A. M. (2011). Chemical composition and therapeutic effects of Lippia alba (Mill.) N. E. Brown leaves hydro-alcoholic extract in patients with migraine. Fitomedicina, 18(14), 1197 - 1201. 10.1016/ j.phymed.2011.06.016.

Coutinho, D. F., Amaral, F. M. M., Luz, T. R. S. A., Brito, M. C. A., Almeida, J. S., Pimentel, K. B. A., Silva, M. V. S. (2020). Prescrição em fitoterapia: orientações para profissionais da área de saúde visando o uso racional de plantas medicinais e fitoterápicos, In: Ciências da Saúde: Avanços Recentes e Necessidades Sociais 2. 2 ed. Ponta Grossa: Atena Editora, 2020, 219 - 252.

Desantana, J. M., \& Perissinotti, D. M. N. (2020). Definição de dor revisada após quatro décadas. Brazilian Journal of Pain, 3 (3), 197 - 198.

Dias, E. C. M., Trevisan, D. D., Nagai, S. C., Ramos, N. A., \& Silva, E. M. (2017). Uso de fitoterápicos e potenciais riscos de interações medicamentosas: reflexões para prática segura. Revista Baiana de Saúde Pública, 41(2), 297 - 307.

Dresch, R. R., Libório, Y. B., Czermainski, S., \& Costa, B. (2021). Compilação de levantamentos de uso de plantas medicinais no Rio Grande do Sul. Physis: Revista de Saúde Coletiva, 31(2), 1 -14.

Galvão, T. F., \& Pereira, M. G. (2014). Revisões sistemáticas da literatura: passos para sua elaboração. Epidemiologia e Serviços de Saúde, $23,183-184$.

Ghavipour, M., Sotoudeh, G., Tavakoli, E., Mowla, K., Hasanzadeh, J., \& Mazloom, Z. (2017). Pomegranate extract alleviates disease activity and some blood biomarkers of inflammation and oxidative stress in Rheumatoid Arthritis patients. European Journal of Clinical Nutrition, $71(1)$, 92 - 96. 10.1038 / ejcn.2016.151. 
Godinho, J. W. L. S. (2014). Atenção Farmacêutica em Fitoterapia: avaliação da comercialização e controle de qualidade de amostras de Passiflora edulis Sims. adquiridas em farmácias e drogarias no município de São Luís, estado do Maranhão. 31f. Monografia (Graduação em Farmácia Bioquímica) Universidade Federal do Maranhão, São Luís, 2014.

Griz, S. A. S., Matos-Rocha, T. J., Santos, A. F., Costa, J. G., \& Mousinho, K. C. (2017). Medicinal plants profile used by the 3rd District population of Maceió-AL. Brazilian Journal of Biology, 77(4),794 - 802. 10.1590 / 1519-6984.01116.

Hasenclever, L., Paranhos, J., Costa, C. R., Cunha, G., \& Vieira, D. (2017). A indústria de fitoterápicos brasileira: desafios e oportunidades. Ciência \& Saúde Coletiva, 22(8), $2559-2569$.

Hashempur, M. H., Sadrneshin, S., Mosavat, S. H., \& Ashraf, A. (2016). Green tea (Camellia sinensis) for patients with knee osteoarthritis: A randomized open-label active-controlled clinical trial. Clinical Nutrition, 37(1), 85 - 90. 10.1016 / j.clnu.2016.12.004.

Heydari, M., Homayouni, K., Hashempur, M. H., \& Shams, M. (2016). Topical Citrullus colocynthis (bitter apple) extract oil in painful diabetic neuropathy: A double-blind randomized placebo-controlled clinical trial. Journal of Diabetes, 8(2), 246 - 252. 10.1111 / 1753-0407.12287.

Hosseini, S. H, Bibak, H. Ghara, A. R, Sahebkar, A, \& Shakeri, A. (2021). Ethnobotany of the medicinal plants used by the ethnic communities of Kerman province, Southeast Iran. Journal of Ethnobiology and Ethnomedicine, 17(1), 31. 10.1186 / s13002-021-00438-z.

IASP. Defending the IASP definition of pain. Monist 2017, 100, 439-464.

Islam, A. T. M. R, Hasan, M, Islam, T., Rahman, A., Mitra, S., \& Das, S. K. (2020). Ethnobotany of Medicinal Plants Used by Rakhine Indigenous Communities in Patuakhali and Barguna District of Southern Bangladesh. Journal of Evidence-based Integrative Medicine, 25, 2515690X20971586. 10.1177 / $2515690 X 20971586$.

Jorim, R. Y., Korape, S., Legu, W., Koch, M., Barrows, L. R., Matainaho, T. K., \& Rai, P. P. (2012). An ethnobotanical survey of medicinal plants used in the eastern highlands of Papua New Guinea. Journal of Ethnobiology and Ethnomedicine, 8 (47). 10.1186 / 1746-4269-8-47.

Kazanci, C., Oruç, S., \& Mosulishvili, M. (2020). Medicinal ethnobotany of wild plants: a cross-cultural comparison around Georgia-Turkey border, the Western Lesser Caucasus. Journal of Ethnobiology and Ethnomedicine, 16 (1) 71. 10.1186 / s13002-020-00415-y.

Kuptniratsaikul, V., Dajpratham, P., Taechaarpornkul, W., Buntragulpoontawee, M., Lukkanapichonchut, P., Chootip, C., Saengsuwan, J., Tantayakom, K., \& Laongpech, S. (2014). Eficacy and safety of Curcuma domestica extracts compared with ibuprofen in patients with knee osteoarthritis: a multicenter study. Clinical Interventions in Aging, 9, 451 - 458. 10.2147 / CIA.S58535.

Kutal, D. H., Kunwar, R. M., Uprety, Y., Adhikari, Y. P., Bhattarai, S., Adhikari, B., Kunwar, L. M., Bhatt, M. D., \& Bussmann, R. W. (2021). Selection of medicinal plants for traditional medicines in Nepal. Journal of Ethnobiology and Ethnomedicine, 17, (1) 59. 10.1186 / s13002-021-00486-5.

Lauche, R., Gräf, N., Cramer, H., Al-Abtah, J., Dobos, G., \& Saha, F. J. (2016). Efficacy of Cabbage Leaf Wraps in the Treatment of Symptomatic Osteoarthritis of the Knee: A Randomized Controlled Trial. The Clinical Journal of Pain, 32, (11), 961 - 971. 10.1097 / AJP.0000000000000352.

Lucena, C. M., Lucena, R. F., Costa, G. M., Carvalho, T. K., Costa, G. G., Alves, R. R., Pereira, D. D., Ribeiro, J. E., Alves, C. A., Quirino, Z. G. \& Nunes, E. N. (2013). Use and knowledge of Cactaceae in Northeastern Brazil. Journal of Ethnobiology and Ethnomedicine, 9, (62). 10.1186/1746-4269-9-62.

Maema, L. P, Potgieter, M, \& Mahlo, S. M. (2016). Nvasive Alien Plant Species Used For The Treatment Of Various Diseases In Limpopo Province, South Africa. African Journal of Traditional, Complementary, and Alternative Medicines, 13, (4), 223 - 231. 10.21010 / ajtcam.v13i4.29.

Magalhães, P. K. A, Araujo, E. N, Santos, A. M, Vanderlei, M. B, Souza, C. C. L, Correia, M. S, Fonseca, S. A, Pavão, J. M. J. S, Souza, M. A, Costa, J. G, Santos, A. F, \& Matos-Rocha, T. J. (2021). Ethnobotanical and ethnopharmacological study of medicinal plants used by a traditional community in Brazil's northeastern. Brazilian Journal of Biology, 82, e2376424. 10.1590 / 1519-6984.237642.

Malik, K., Ahmad, M., Zafar, M, Ullah, R., Mahmood, H. M., Parveen, B., Rashid, N., Sultana, S., Shah, S. N., \& Lubna. (2019). An ethnobotanical study of medicinal plants used to treat skin diseases in northern Pakistan. BMC Complementary and Alternative Medicine, 19, (1), 210. 10.1186/s12906-019-2605-6.

Marchi, J. P., Tedesco, L., Melo, A. Da C., Frasson, A. C., França, V. F., Sato, S. W., \& Lovato, E. C. W. (2016). Curcuma longa L.:o açafrão da terra, e seus benefícios medicinais. Arquivos de Ciências da Saúde da UNIPAR, 20, (3), 189-194.

Marques, M. A. A., Lima, D. A., Andreotti, C. E., Gasparotto Junior, A., \& Lourenço, E. L. B. (2016). Caracterização das plantas medicinais e medicamentos fitoterápicos para tratamento da osteoporose utilizados no Brasil. Arquivos de Ciências da Saúde, 20(3), 183 - 188.

Medeiros, M. F., \& De Albuquerque, U. P. (2012). The pharmacy of the Benedictine monks: the use of medicinal plants in Northeast Brazil during the nineteenth century (1823-1829). Journal of Ethnopharmacology, 139(1), 280 - 286. 10.1016 / j.jep.2011.11.014.

Medeiros, P. M., Figueiredo, K. F., Gonçalves, P. H. S., Caetano, R. A., Santos, É. M. D. C., Dos Santos, G. M. C., Barbosa, D. M., De Paula, M., \& Mapeli, A. M. (2021). Wild plants and the food-medicine continuum-an ethnobotanical survey in Chapada Diamantina (Northeastern Brazil). Journal of Ethnobiology and Ethnomedicine, 17, (1), 37. 10.1186/ s13002-021-00463-y.

Miguéis, G. D. S., Da Silva, R. H., Damasceno Júnior, G. A., \& Guarim-Neto, G. (2019). Plants used by the rural community of Bananal, Mato Grosso, Brazil: Aspects of popular knowledge. Plos One, 14, 1, e0210488. 10.1371 / journal.pone.0210488.

Mongalo, N. I., \& Makhafola, T. J. (2018). Ethnobotanical knowledge of the lay people of Blouberg area (Pedi tribe), Limpopo Province, South Africa. Journal of Ethnobiology and Ethnomedicine, 14(1), 46. 10.1186 / s13002-018-0245-4.

Montesano, V. Negro, D., Sarli, G., De Lisi, A., Laghetti, G., \& Hammer, K. (2012). Notes about the uses of plants by one of the last healers in the Basilicata region (South Italy). Journal of Ethnobiology and Ethnomedicine, 8, 15. 10.1186 / 1746-4269-8-15. 
Mozaffari-Khosravi, H., Naderi, Z., Dehghan, A., Nadjarzadeh, A., \& Fallah Huseini, H. (2016). Effect of Ginger Supplementation on Proinflammatory Cytokines in Older Patients with Osteoarthritis: Outcomes of a Randomized Controlled Clinical Trial. Journal of Nutrition in Gerontology and Geriatrics, 35(3), p. 209 - 218. 10.1080 / 21551197.2016.1206762.

Musharraf, M. U., Ahmad, Z., \& Yaqub, Z. (2017). Comparison of topical capsaicin and topical turpentine Oil for treatment of painful diabetic neuropathy. Journal of Ayub Medical College, Abbottabad: JAMC, 29, (3), 384 - 387.

Nash, R. J., Azantsa, B. K., Sharp, H., \& Shanmugham, V. (2018). Effectiveness of Cucumis sativus extract versus glucosamine-chondroitin in the management of moderate osteoarthritis: a randomized controlled trial. Clinical Interventions in Aging, 13, 2119 - 2126. 10.2147 / CIA.S173227.

Nasiri, A., Mahmodi, M. A., \& Nobakht, Z. (2016). Effect of aromatherapy massage with lavender essential oil on pain in patients with osteoarthritis of the knee: A randomized controlled clinical trial. Complementary Therapies in Clinical Practice, 25, 75 - 80. 10.1016 / j.ctcp.2016.08.002.

Nicoletti, M. A., Carvalho, K. C., Oliveira Jr, M. A., Bertasso, C. C., Caporossi, P. Y., \& Tavares, A. P. L. (2010). Popular use of medicines containing drugs from vegetal source and/or medicinal plants: main interactions resulting from that. Revista Saúde, 4(1), 25 - 39.

Nicoletti, M. A., Oliveira-Júnior, M. A., Bertasso, C.C., Caporossi, P. Y., \& Tavares, A. P. L. (2007).Principais interações no uso de medicamentos fitoterápicos. Infarma, 19, (1/2), $32-40$.

Niempoog, S., Pawa, K. K., \& Amatyakul, C. (2012). The Efficacy of Powdered Ginger in Osteoarthritis of The Knee. The Journal of Medical Association of Thailand, 95(1), 59. http://jmatonline.com/index.php/jmat/article/view/1904/0.

Novotna, B., Polesny, Z., Pinto-Basto, M. F., Van Damme, P., Pudil, P., Mazancova, J., \& Duarte, M. C. (2020). Medicinal plants used by 'root doctors', local traditional healers in Bie province, Angola. Journal of Ethnopharmacology, 26. https://doi.org/10.1016/j.jep.2020.112662.

Oliveira, F. C., Silveira, P. F., Bandeira, M. A. M., \& Arrais, P. S. D. (2008). Farmacovigilância e reações adversas às plantas medicinais e fitoterápicos: uma realidade. Revista Brasileira de Farmacognosia, 18, 618 - 626.

Oliveira, F. C., Albuquerque, U. P., Fonseca-Krueliv, V. S., \& Hanazaki, N. (2009). Avanços nas pesquisas etnobotânicas no Brasil. Acta Botanica Brasilica, $23(2), 590-605$.

Pedrollo, C. T, Kinupp, V. F, Shepard, G. Jr, \& Heinrich, M. (2016). Medicinal plants at Rio Jauaperi, Brazilian Amazon: Ethnobotanical survey and environmental conservation. Journal of Ethnopharmacology, 186, 111-124. 10.1016 / j.jep.2016.03.055.

Pereira, J. B. A., Rodrigues, M. M., Morais, I. R., Vieira, C. R. S., Sampaio, J. P. M., Moura, M. G., Damasceno, M. F. M., Silva, J. N., Calou, I. B. F., Deus, F. A., Peron, A. P, Abreu, M. C., Militão, G. C. G., \& Ferreira, P. M. P. (2015). O papel terapêutico do Programa Farmácia Viva e das plantas medicinais. Revista Brasileira de Plantas Medicinais, 17(4), 550 - 561.

Posso, I. P., Palmeira, C. C. A., \& Vieira, E. B. M. (2016). Epidemiology of neuropathic pain. Revista Dor, 17, S11-14.

Quintans, J.S., Antoniolli, Â.R., Almeida, J.R., Santana-Filho, V. J., \& Quintans-Júnior, L.J. (2014). Natural Products Evaluated in Neuropathic Pain Models A Systematic Review. Basic \& Clinical Pharmacology \& Toxicology, 114, $442-450$.

Quiroga, R, Meneses, L, \& Bussmann, R. W. (2012). Etnobotânica medicinal em Huacareta (Chuquisaca, Bolívia). Journal of Ethnobiology and Ethnomedicine,8(29). 10.1186 / 1746-4269-8-29.

Rahmatullah, M., Khatun, Z., Hasan, A., Parvin, W., Moniruzzaman, M., Khatun, A., Mahal, M. J., Bhuiyan, M. S., Mou, S. M., \& Jahan, R. (2012). Survey and scientific evaluation of medicinal plants used by the Pahan and Teli tribal communities of Natore district, Bangladesh. African Journal of Traditional, Complementary, and Alternative Medicines: AJTCAM, 9(3) 366 - 373. 10.4314 / ajtcam.v9i3.10.

Raja, S.N., Carr, D.B., Cohen, M., Finnerup, N. B., Flor, H., Gibson, S., Keefeh, F. J., Mogili , J. S., Ringkampj, M., Slukak , K. A., Songl , X., Stevensm, B., Sullivann, M. D., Tutelmano , P. R., Ushidap , T., \& Vaderq, K. (2020). The revised International Association for the Study of Pain definition of pain: $\begin{array}{llll}\text { concepts, } & \text { challenges, } & \text { compromises. } & \text { Pain. }\end{array}$ content/uploads/2020/07/The_revised_International_Association_for_the.98346.pdf.

Rakotoarivelo, N. H., Rakotoarivony, F., Ramarosandratana, A. V. Jeannoda, V. H., Kuhlman, A. R., Randrianasolo, A., \& Bussmann, R. W. (2015). Medicinal plants used to treat the most frequent diseases encountered in Ambalabe rural community, Eastern Madagascar. Journal of Ethnobiology and Ethnomedicine, 11(68). 10.1186 / s13002-015-0050-2.

Rudrappa, G. H., Chakravarthi, P. T., \& Benny, I. R. (2020). Efficacy of high-dissolution turmeric-sesame formulation for pain relief in adult subjects with acute musculoskeletal pain compared to acetaminophen: A randomized controlled study. Medicine, 99(28), e20373. 10.1097 / MD.0000000000020373.

Santos, M. R. A., Lima, M. R., \& Oliveira, C. L. L. G. (2014). Medicinal plants used in Rondônia, Western Amazon, Brazil / Plantas medicinais usadas em Rondônia, Amazônia Ocidental, Brasil. Revista Brasileira de Plantas Medicinais, 16(3) 707 - 720.

Shep, D., Khanwelkar, C., Gade, P., \& Karad, S. (2020). Efficacy and safety of combination of curcuminoid complex and diclofenac versus diclofenac in knee osteoarthritis: A randomized trial. Medicine, 99(16), e19723. 10.1097 / MD.0000000000019723.

Shoara R, Hashempur Mh, Ashraf A, Salehi A, Dehshahri S, \& Habibagahi Z. (2015). Efficacy and safety of topical Matricaria chamomilla L. (chamomile) oil for knee osteoarthritis: A randomized controlled clinical trial. Complementary Therapies in Clinical Practice, 21(3), 181 - 187. 10.1016 / j.ctcp.2015.06.003.

Silva, A. G., Machado, E. R., Almeida, L. M., Nunes, R. M., Giesbrecht, P. C., Costa, R. M., Costa, H. B., Romão, W., \& Kuster, R. M. A (2015). Clinical Trial with Brazilian Arnica (Solidago chilensis Meyen) Glycolic Extract in the Treatment of Tendonitis of Flexor and Extensor Tendons of Wrist and Hand. Phytotherapy Research: PTR, 29(6), 864 - 869. 10.1002 / ptr.5323. 
Silva, O. N., Amaral, F. M. M., Godinho, J. W. L.S., Ferreira, T. T. D., Coutinho, D. F., Neiva, V. A., Neiva Neto, R. R., \& Bastos, W. M. (2021). Toxicidade de plantas de uso medicinal: desmitificando o "se natural, não faz mal". In: Trajetória e pesquisa nas ciências farmacêuticas. 1 ed. Ponta Grossa: Atena Editora, 1, $11-32$.

Simões, C. O. M., Schenkel, E. P., Mello, J. C. P., Mentz, L. A., \& Petrovick, P. R. (2017). Farmacognosia: do produto natural ao medicamento. 1. ed. Porto Alegre: Artmed, 486.

Singh, A. G., Kumar, A., \& Tewari, D. D. (2012). An ethnobotanical survey of medicinal plants used in Terai forest of western Nepal. Journal of Ethnobiology and Ethnomedicine, 8, 19. 10.1186/ 1746 - 4269-8-19.

Singla, R. K., Guimarães, A. G., Zengin, G. (2021). Application of plant secondary metabolites to pain neuromodulation. Frontiers in Pharmacology, 14. https://doi.org/10.3389/fphar.2020.623399.

Silveira, P. F., Bandeira, M. A. M., \& Arrais, P. S. D. (2008). Farmacovigilância e reações adversas às plantas medicinais e fitoterápicos: uma realidade. Revista Brasileira de Farmacognosia, 18, 618-626.

Soo-May, L., Sanip, Z., Ahmed-Shokri, A., Abdul-Kadir, A. M. D., \& Lazin, M. R. (2018). The effects of Momordica charantia (bitter melon) supplementation in patients with primary knee osteoarthritis: A single-blinded, randomized controlled trial. Complementary Therapies in Clinical Practice, 32 , 181 - 186. $10.1016 /$ j.ctcp.2018.06.012

Souza, G. R., Silva, J. C, Oliveira Júnior, R. G, Lima-Saraiva, S. R. G., Guimarães, A. L., Oliveira, A. P, \& Almeida, J. R. G. S. (2015). Atividade antinociceptiva do extrato etanólico das folhas de Morus nigra L. (Moraceae). Revista de Ciências Farmacêuticas Básica e Aplicada, $36(1), 137$ - 142.

Tabuti, J. R., Kukunda, C. B., Kaweesi, D., \& Kasilo, O. M. (2012). Herbal medicine use in the districts of Nakapiripirit, Pallisa, Kanungu, and Mukono in Uganda. Journal of Ethnobiology and Ethnomedicine, 8(35). 10.1186 / 1746-4269-8-35.

Tovar, R. T., \& Petzel, R. M. (2009). Herbal toxicity. Disease-a-month: DM, 55(10), 592 - 641. doi.org/10.1016/j.disamonth.2009.05.001.

Tugume, P., Kakudidi, E. K., Buyinza, M., Namaalwa, J., Kamatenesi, M., Mucunguzi, P., \& Kalema, J. (2016). Ethnobotanical survey of medicinal plant species used by communities around Mabira Central Forest Reserve, Uganda. Journal of Ethnobiology ond Ethnomedicine, 12, (5). 10.1186 / s13002-0150077-4.

Tugume, P., \& Nyakoojo, C. (2019). Ethno-pharmacological survey of herbal remedies used in the treatment of pediatric diseases in Buhunga parish, Rukungiri District, Uganda. BMC Complementary and Alternative Medicine, 19(1), 353. doi.org/10.1186/s12906-019-2763-6.

Uddin, M. S., Mamun, A. A., Sumsuzzman, D. M., Ashraf, G. M., Perveen, A., Bungau, S. G., Mousa, S. A., El-Seedi, H. R., Bin-Jumah, M. N., \& AbdelDaim, M. M. (2020). Emerging promise of cannabinoids for the management of pain and associated neuropathological alterations in Alzheimer's disease. Frontiers in Pharmacology, 11, 1097. https://doi.org/10.3389/fphar.2020.01097.

Vasconcelos, F. H., \& Araújo, G. C. (2018). Prevalência de dor crônica no Brasil: estudo descritivo. Brazilian Journal of Pain, 1 (2), 176 -179.

Wang, Z., Jones, G., Winzenberg, T., Cai., G, Laslett, L. L., Aitken, D., Hopper, I., Singh, A., Jones, R., Fripp, J., Ding, C., \& Antony, B. (2020). Effectiveness of Curcuma longa Extract for the Treatment of Symptoms and Effusion-Synovitis of Knee Osteoarthritis: A Randomized Trial. Annals of Internal Medicin, 173(11), 861-869. 10.7326/M20-0990. Epub 2020 Sep 15. PMID: 32926799

World Health Organization. WHO (1999). Monographs on selected medicinal plants. Geneva: World Health Organization. 1, 295.

World Health Organization. WHO. (2002). Traditional Medicine: Growing Needs and Potential. 2, 357. 